\title{
Managing head and neck cancer patients with tracheostomy or laryngectomy during the COVID-19 pandemic
}

\author{
Maxwell P. Kligerman MD, MPH ${ }^{1,2}$ () | Neelaysh Vukkadala MD ${ }^{1,2}$ | \\ Raymond K. Y. Tsang FRCS Ed (ORL) MS ${ }^{3} \odot ｜$ John B. Sunwoo MD ${ }^{1}$ \\ F. Christopher Holsinger MD ${ }^{1}$ ( ) | Jason Y. K. Chan FRCS Ed (ORL) ${ }^{4}$ । \\ Edward J. Damrose MD, MPH ${ }^{1,2}$ ｜ Ann Kearney CScD CCC-SLP, BCS-S ${ }^{2}$ | \\ Heather M. Starmer MA, CCC-SLP, BCS-S ${ }^{1}$
}

\author{
${ }^{1}$ Division of Head and Neck Surgery, \\ Stanford University, Palo Alto, California \\ ${ }^{2}$ Division of Laryngology, Department of \\ Otolaryngology, Stanford University, \\ Palo Alto, California \\ ${ }^{3}$ Department of Otorhinolaryngology- \\ Head and Neck Surgery, The Chinese \\ University of Hong Kong, Shatin, \\ NT East, Hong Kong \\ ${ }^{4}$ Division of Otorhinolaryngology-Head \\ and Neck Surgery, Department of Surgery, \\ The University of Hong Kong, \\ Pok Fu Lam, Hong Kong \\ Correspondence \\ Maxwell P. Kligerman, Division of Head \\ and Neck Surgery, Stanford University, \\ Palo Alto, California, USA. \\ Email: maxwell3@stanford.edu
}

\begin{abstract}
Head and neck cancer patients with tracheostomies and laryngectomies, as well as their healthcare providers, face unique challenges in the context of the current COVID-19 pandemic. This document consolidates best available evidence to date and presents recommendations to minimize the risks of aerosolization and SARS-CoV-2 exposures in both the inpatient and outpatient settings. The cornerstones of these recommendations include the use of closed-circuit ventilation whenever possible, cuffed tracheostomy tubes, judicious use of heat moisture exchange units, appropriate personal protective equipment for providers and patients, meticulous hand hygiene, and minimal manipulation of tracheostomy tubes.
\end{abstract}

\section{K E Y W O R D S}

Aerosolization, COVID-19, Laryngectomy, SARS-CoV-2, Tracheostomy

\section{1 | INTRODUCTION}

The COVID-19 pandemic has created unprecedented new challenges for the health care system, including a unique set of challenges for patients with head and neck cancer. Although multiple prior studies have outlined indications and risk mitigation strategies for tracheotomy in patients with COVID-19, there remains no clear consensus for inpatient and outpatient management of head and neck cancer patients with tracheostomy and/or total laryngectomy (TL) during the pandemic. ${ }^{1-3}$ Given the highly contagious nature of the virus from respiratory droplets and aerosolization, ${ }^{4}$ patients with tracheostomy and TL have a particularly high risk of transmission to others and merit special attention in terms of strategies to minimize viral spread.
Although elective surgery across the United States has come to a halt, urgent and emergent tracheostomy and TL continue for patients with head and neck cancer. ${ }^{5}$ Unlike in patients with traditional acute respiratory distress syndrome, practices emerging from China where the pandemic began suggest that long-term intubation alone should not be a justification for tracheostomy in SARSCoV-2-positive patients. ${ }^{6}$ However, there are tens of thousands of head and neck cancer patients with long-standing tracheostomy and TL who continue to require medical care in both the inpatient and outpatient contexts. ${ }^{7}$ This commentary compiles the best available evidence to provide recommendations aimed at minimizing transmission of COVID-19 when caring for head and neck cancer patients with tracheostomies and TL. Policies are bound to 
vary by institution and specific patient requirements and available resources. Global transparent communication may help to reduce risk to health care workers and improve outcomes for individual patients and societies facing this unprecedented pandemic.

\section{2 | RISK OF AEROSOLIZATION AND DROPLET FORMATION IN PATIENTS WITH TRACHEOSTOMY}

COVID-19 can spread by direct contact, droplet, and likely also aerosolization. ${ }^{4,8,9}$ Aerosolization refers to the process by which small particles are formed and dispersed in the air. ${ }^{10}$ It is important to distinguish aerosolization from droplet formation, which refers to the process by which fluids in the form of large droplets come into direct contact with another person thereby causing infection. In contrast to droplets, aerosolized SARS-CoV-2 does not require personal contact with the source patient and has been shown to remain viable in the air for upwards of 3 hours. ${ }^{4}$ For this reason, procedures or patient groups with increased risk of aerosolization may significantly increase viral transmission. ${ }^{11,12}$ Some common procedures known to lead to aerosolization include intubation, tracheotomy, airway suctioning, nebulizer treatments, manipulation of oxygen masks, and noninvasive positive pressure ventilation. ${ }^{13,14}$

It remains unclear exactly how much and how often patients with tracheostomy and TL are aerosolizing. The amount likely differs based on unique patient factors. Based on data from the 2013 SARS-CoV-1 outbreak and early data from the current COVID-19 pandemic, it is likely that patients with fresh tracheostomy or TL are at significantly higher risk of spreading viral particles compared to other patients given high rates of aerosolizing procedures and equipment, including suctioning requirements, nebulizer treatments, open stomas, and open humidification with trach collar. ${ }^{11,15,16}$ In addition to the risk of aerosolization, there is also an increased risk of droplet formation given frequent productive coughing in recent surgical patients. Given the increased risk of aerosolization and droplet formation in conjunction with the high rates of undocumented infections and asymptomatic carriers, precautions for all patients with tracheostomy and TL may be indicated. ${ }^{17}$

\section{3 | RISK REDUCTION STRATEGIES}

Flow charts of strategies aimed at minimizing risk of transmission are shown in Figures 1 and 2. The cornerstones of these recommendations include the use of closed-circuit ventilation whenever possible, cuffed tracheostomy tubes, judicious use of heat moisture exchange units (HMEs) as tolerated, appropriate personal protective equipment (PPE) for providers and patients at all times, and minimal manipulation of tracheostomy tubes. These strategies are described in the following section in more detail.

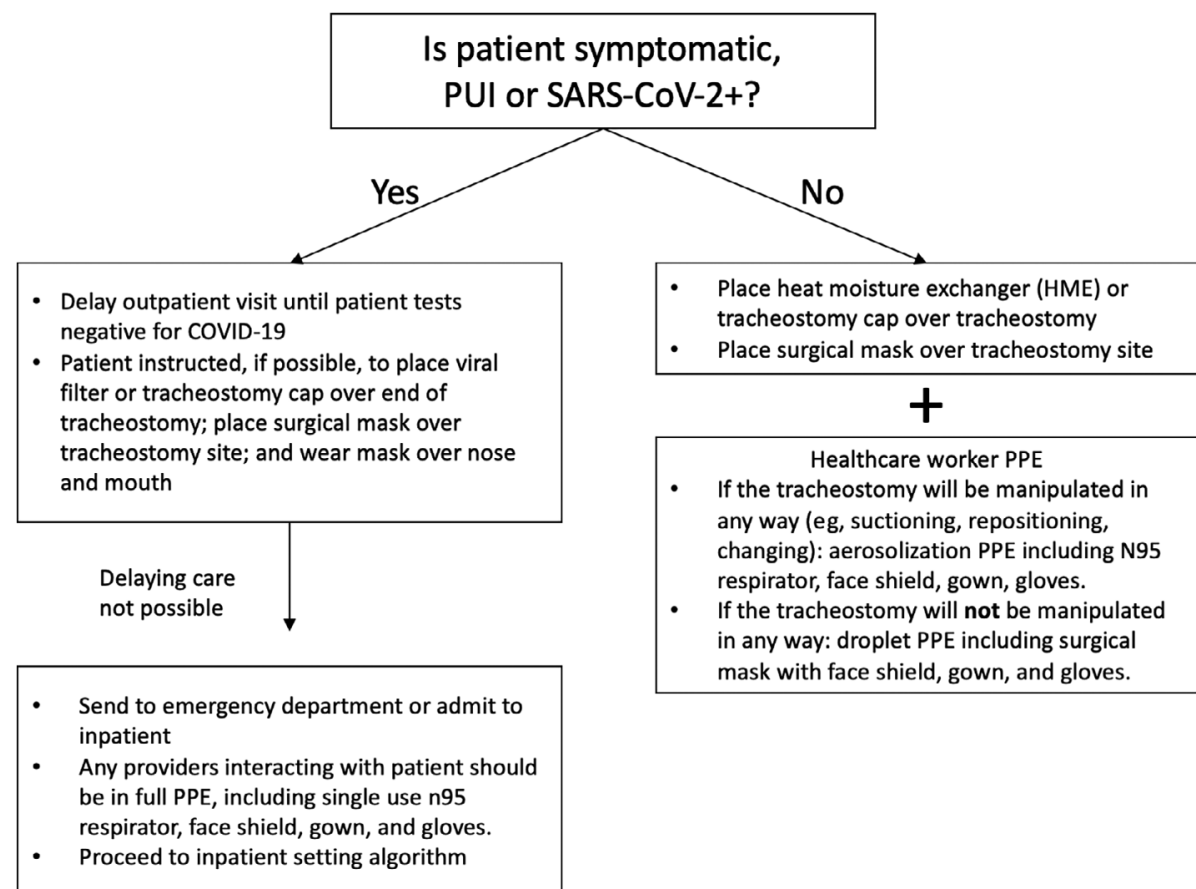

F I G URE 1 Outpatient guidelines for patients with tracheostomies or laryngectomies 
F I G U RE 2 Inpatient guidelines for patients with tracheostomies or laryngectomies

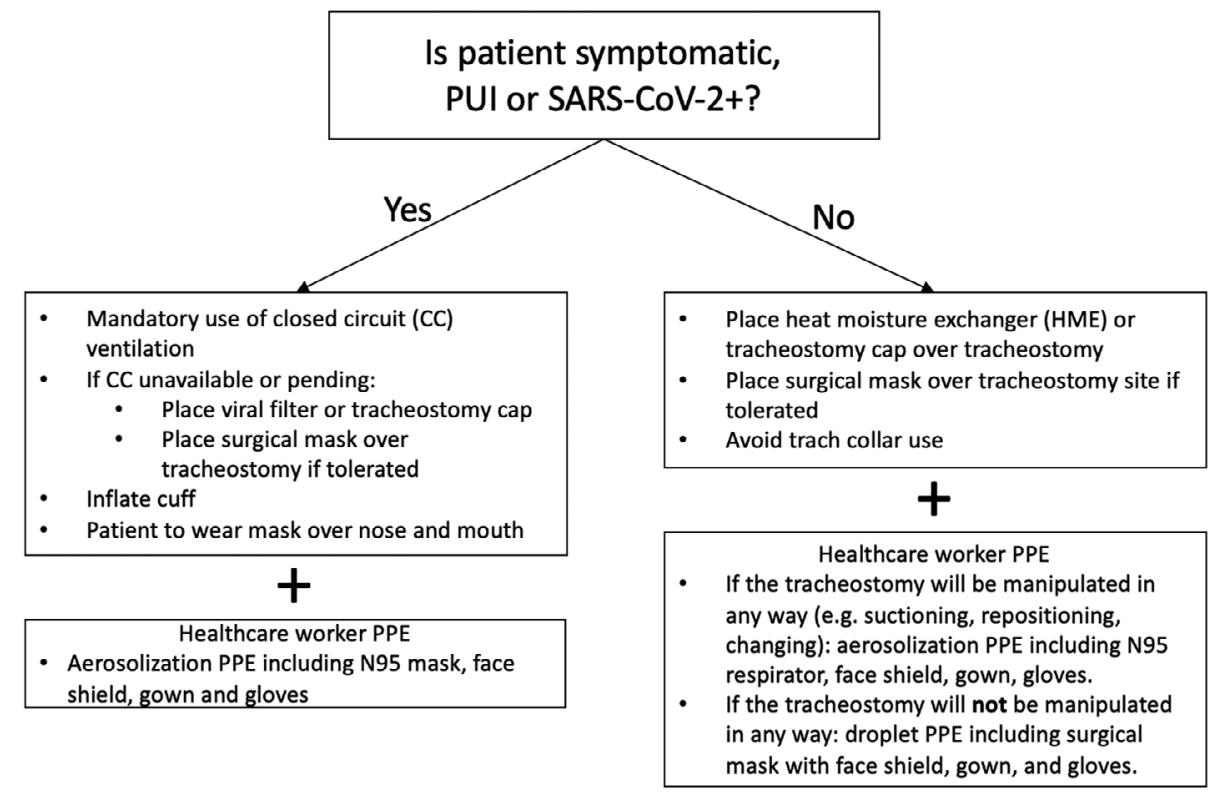

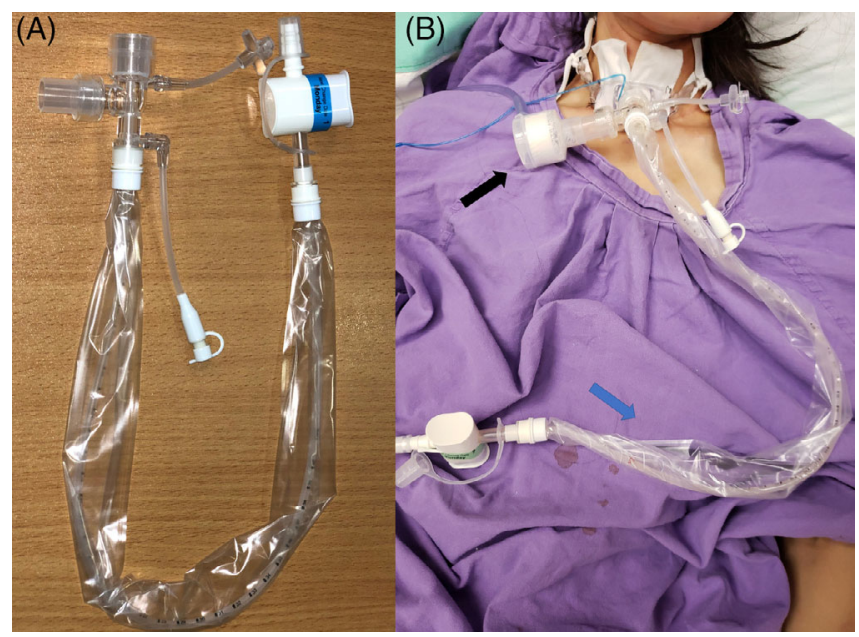

F I G U RE 3 Example configuration for in-line suction without positive pressure ventilation. Figure A demonstrates a two-valve adapter; Figure B demonstrates an HME on one valve (black arrow) and suction on the other (blue arrow) [Color figure can be viewed at wileyonlinelibrary.com]

\section{1 | Closed circuit ventilation with in-line suction}

Closed circuit ventilation, particularly in cases of known or suspected COVID-19, may decrease the risk of aerosolization and droplet formation. ${ }^{1}$ Cuffed tracheostomy tubes should be used in these circumstances to decrease leaks in the circuit. Use of in-line suction, even for patients who may not require chronic closed-circuit ventilation, may also decrease risk of aerosolization and droplets (Figure 3). ${ }^{17}$ Nebulizer treatments should be avoided or used with caution as well to minimize risk of aerosolization. ${ }^{11}$

\subsection{Heat moisture exchange units}

Heat moisture exchange units (HMEs) may reduce both aerosolization and droplet formation. Whenever tolerated, HMEs should be used instead of open tracheostomy collar with humidified air. Atos Medical has recently developed the Provox Micron HME filter that may be a helpful option for patients with suspected or confirmed COVID-19. While efficacy for COVID-19 has not specifically been tested, previous viral filtration efficacy was demonstrated to be $99 \%{ }^{18}$ Although the Micron was initially designed for patients following laryngectomy, it may be used with a 15-mm hub adaptor for patients with tracheostomy. Standard HMEs without viral filtration may be used for patients without known infection or symptoms. Cuffed tracheostomy tubes are ideal for SARS-CoV-2-positive patients as they may decrease leak around the HME and tracheostomy tube and can decrease airflow through the oropharynx and nasopharynx. However, these potential benefits must be balanced against the increased suction requirements while the cuff is up as patients will be less able to self-clear secretions.

\section{3 | Appropriate use of PPE}

PPE is essential to mitigate the risk of transmission. ${ }^{19,20}$ Proper fit testing is essential for effective filtration with N95 masks. For all patients with tracheostomy/TL who are known to be SARS-CoV-2 positive or under investigation, the clinician should wear appropriate aerosolization PPE, including at a minimum an N95 mask, face shield, gown, and gloves at all times. For patients who are asymptomatic and/or SARS-CoV-2 negative, providers should at 
minimum wear droplet precaution PPE, including a surgical mask with face shield, gown, and gloves at all times while in the room. If there are any procedures that may lead to aerosolization (ie, trach change and/or suctioning without an inline suction), then the provider should wear aerosolization PPE, including an N95 respirator, regardless of patient SARS-CoV-2 status due to unknown falsenegative COVID PCR testing rates at this time and high rates of asymptomatic carriers. Removal of PPE after exposure to an individual infected with COVID is one of the highest risk periods for contamination. ${ }^{21}$ Apart from provision of PPE, prior training of proper gown up and gown down of PPE must be provided to all health care workers involved in caring for patients with suspected or confirmed SARS-CoV-2. ${ }^{22}$ All staff should be vigilant in adhering to the gown up and gown down protocol.

Patients should also wear PPE to decrease risk of transmission. Any patient known to be SARS-CoV-2 positive or under investigation should wear a surgical mask over nose and mouth, regardless of tracheostomy/TL status. Patients not on a closed ventilation circuit should also wear a surgical mask over their stoma if tolerated as this may decrease spread of droplets from leakage around the stoma and/or HME. Any SARS-CoV-2-negative or asymptomatic patients who are immunocompromised (ie, patients receiving active chemotherapy, active radiation, active immunotherapy, with a history of lung transplant, less than 1 year status post solid organ or bone marrow transplant, or with neutropenia) should wear a mask over the nose and mouth at all times while in the health care setting to decrease their own risk of becoming infected.

\section{4 | Hygiene}

Although the importance of hand hygiene to minimize transmission is well established, patients with tracheostomy and TL have some special considerations. Apart from acquiring the virus from inhalation of droplets infected with the virus, tracheostomy and TL patients may become infected if their hands contaminated with the virus touch the respiratory mucosa of the tracheostoma. Thorough hand hygiene should be performed immediately prior to and following any contact with the tracheostomy or laryngectomy stoma. Patients who use a tracheoesophageal voice prosthesis (TEP) should use an HME interface for voicing to minimize hand to stoma contact. Patients using an electrolarynx should clean their device frequently with disinfectant wipes and those using a pneumatic tube or Taiwan tube artificial larynx should clean the device with hydrogen peroxide following each use. ${ }^{23}$

\section{5 | Minimize exposures and procedures}

All nonurgent procedures that can be reasonably delayed (ie, elective tracheostomy tube change or TEP exchange) should be postponed and manipulation of the tracheostomy and/or laryngectomy site should be minimized. TEP leakage may be managed by the temporary use of a plug or thickened liquids when possible. Similarly, all nonurgent clinic visits should be postponed and/or converted to telehealth visits when possible. The absolute minimum number of providers required to safely care for and evaluate each patient should be used.

\section{6 | SARS-CoV-2 screening}

When PCR testing for the virus becomes more widely available in the surgeon's practice, preoperative testing for all patients scheduled for tracheostomy and/or TL should be strongly considered. There may also be a role for SARS-CoV2 testing for patients with tracheostomy or TL who are admitted to the inpatient setting for an extended period of time, but this may vary based on the individual patient's risk, hospital setting, and other considerations such as endemic risk.

\section{4 | CONCLUSIONS}

Head and neck cancer patients with tracheostomy and TL as well as their health care providers face unique challenges in the context of the current COVID-19 pandemic. Although practices and recommendations are bound to evolve over time, this document may serve as a foundation for future efforts to help minimize the risk of aerosolization and SARS-CoV-2 exposures.

\section{ORCID}

Maxwell P. Kligerman (i) https://orcid.org/0000-00023818-0460

Raymond K. Y. Tsang (1D https://orcid.org/0000-00031956-5821

F. Christopher Holsinger (10) https://orcid.org/0000-00027885-4327

Jason Y. K. Chan (1) https://orcid.org/0000-0002-94804637

\section{REFERENCES}

1. Harrison LR, J; Winter, S; Rocke, J; Heward, E. Guidance for surgical tracheostomy and tracheostomy tube change during the COVID-19 pandemic. 2020. https://www.entuk.org/tracheostomyguidance-during-covid-19-pandemic. Accessed April 2, 2020. 
2. Parker NSB, Fritz M, Rapoport S, et al. Tracheotomy Recommendations During the COVID-19 Pandemic. Am Acad Otolaryngol Head Neck Surg. 2020. https://www.entnet.org/content/ tracheotomy-recommendations-during-covid-19-pandemic.

3. Tay JK, Khoo ML, Loh WS. Surgical considerations for tracheostomy during the COVID-19 pandemic: Lessons learned from the severe acute respiratory syndrome outbreak. JAMA Otolaryngol Head Neck Surg. 2020. https://doi.org/10.1001/jamaoto.2020. 0764.

4. van Doremalen N, Bushmaker T, Morris DH, et al. Aerosol and surface stability of SARS-CoV-2 as compared with SARS-CoV-1. N Engl J Med. 2020;382:1564-1567.

5. Vukkadala N, Qian ZJ, Holsinger FC, Patel ZM, Rosenthal E. COVID-19 and the otolaryngologist - preliminary evidencebased review. Laryngoscope. 2020. https://doi.org/10.1002/lary. 28672.

6. Cui C, Yao Q, Zhang D, et al. Approaching otolaryngology patients during the COVID-19 pandemic. Otolaryngol Head Neck Surg. 2020. https://www.emtnet.org/sites/default/files/ uploads/2_liu_approaching_otolaryngology_patients_during the_covid-19_pandemic.pdf.

7. Brook I. The Laryngectomee Guide. 2013.

8. How COVID-19 Spreads. 2020; https://www.cdc.gov/coronavirus/ 2019-ncov/prevent-getting-sick/how-covid-spreads.html. Accessed April 2, 2020.

9. National Research Council. Rapid Expert Consultation on the Possibility of Bioaerosol Spread of SARS-CoV-2 for the COVID-19 Pandemic. Washington, DC: The National Academies Press; 2020.

10. Tang JW, Settles GS. Images in clinical medicine. Coughing and aerosols. New Engl J Med. 2008;359(15):e19.

11. Tran K, Cimon K, Severn M, Pessoa-Silva CL, Conly J. Aerosol generating procedures and risk of transmission of acute respiratory infections to healthcare workers: A systematic review. PLoS One. 2012;7(4):e35797. https://doi.org/10.1371/journal. pone.0035797.

12. Patel ZM, Fernandez-Miranda J, Hwang PH, et al. Precautions for endoscopic transnasal skull base surgery during the COVID-19 pandemic. Neurosurgery. 2020;nyaa125. https://doi. org/10.1093/neuros/nyaa125.

13. UW Medicine COVID-19 Resource Site-Minimizing Aerosolizing Procedures. 2020.

14. Balakrishnan KSS, Hogikyan N, et al. COVID-19 pandemic: What every otolaryngologist-head \& neck surgeon needs to know for safe airway management. Otolaryngol Head Neck Surg. 2020. https://doi.org/10.1177/0194599820919751.

15. Wei WI, Tuen HH, Ng RWM, Lam LK. Safe tracheostomy for patients with severe acute respiratory syndrome. Laryngoscope. 2003;113(10):1777-1779.

16. Givi B, Schiff BA, Chinn SB, et al. Safety recommendations for evaluation and surgery of the head and neck during the COVID-19 pandemic. JAMA Otolaryngol Head Neck Surg. 2020. https://doi.org/10.1001/jamaoto.2020.0780.

17. Chan JYK, Wong EWY, Lam W. Practical aspects of otolaryngologic clinical services during the 2019 novel coronavirus epidemic: An experience in Hong Kong. JAMA Otolaryngol Head Neck Surg. 2020. https://doi.org/10.1001/jamaoto.2020. 0488 .

18. 7248 Provox Micron HME, Lot: 0709146. Nelson Laboratories; 23 Oct 2014.

19. Loeb M, McGeer A, Henry B, et al. SARS among critical care nurses, Toronto. Emerg Infect Dis. 2004;10(2):251-255.

20. Seto WH, Tsang D, Yung RWH, et al. Effectiveness of precautions against droplets and contact in prevention of nosocomial transmission of severe acute respiratory syndrome (SARS). Lancet (London, England). 2003;361(9368):1519-1520.

21. Lim SM, Cha WC, Chae MK, Jo IJ. Contamination during doffing of personal protective equipment by healthcare providers. Clinic Exp Emerg Med. 2015;2(3):162-167.

22. Zuo M-Z, Huang Y-G, Ma W-H, et al. Expert recommendations for tracheal intubation in critically ill patients with noval coronavirus disease 2019. Chinese Med Sci J. 2020. https://doi.org/ 10.24920/003724.

23. Chalstrey SE, Bleach NR, Cheung D, van Hasselt CA. A pneumatic artificial larynx popularized in Hong Kong. J Laryngol Otol. 1994;108(10):852-854.

How to cite this article: Kligerman MP, Vukkadala N, Tsang RKY, et al. Managing head and neck cancer patients with tracheostomy or laryngectomy during the COVID-19 pandemic. Head \& Neck. 2020;42:1209-1213. https://doi.org/ 10.1002/hed.26171 